\title{
A Clinical Comparative Study of Two Different Materials Used in Recording Neutral Zone in Completely Edentulous Patients at Jaipur Dental College, Jaipur
}

\author{
Nihit Singh Rana ${ }^{1}$ \\ 1Department of Prosthodontics, Jaipur Dental College, Jaipur, Rajasthan, India.
}

\section{ABSTRACT}

\section{BACKGROUND}

Stability of mandibular dentures in highly resorbed ridges is a great challenge and one of the deciding factors in the failure or success of the complete denture. The neutral zone technique is an alternative approach for the construction of complete dentures with such kind of challenges. The objective of this research was to study the accuracy of reproducibility of Neutral Zone by two commonly available dental materials, tissue conditioner and zinc oxide eugenol paste.

\section{METHODS}

Viscogel tissue conditioner and zinc oxide eugenol paste are easy flowing recording materials and can be recorded by using support. Hence to study the accuracy of the reproducibility of the neutral zone, specially designed acrylic rims with occlusal stops were used which supported the recording materials adequately in the posterior region of the inter ridge space. Swallowing method was used to record the neutral zone, five times by each material. Bucco-lingual dimension of the neutral zone was recorded at predetermined reference points for all individuals with the help of a micrometer. 40 patients were selected with Class 1 jaw relations.

\section{RESULTS}

In this in vivo study done in selected 40 patients, selection done according to selection criteria to compare the accuracy of reproducibility of Neutral Zone by tissue conditioner [Dentsply, Visco-gel, Temporary Soft Denture Liner]and zinc oxide eugenol impression paste [DPI Impression Paste ${ }^{\mathrm{R}}$ ]. Neutral zone were recorded in all the cases with both materials and data recorded to create the results. By summarizing the results as width (in mm.) of the neutral zone at point $1,2,3$, and 4 for tissue conditioner [Dentsply, Visco-gel, and Temporary Soft Denture Liner] were $10.36,10.06,10.51$ and 10.21 respectively. For zinc oxide eugenol impression paste [DPI Impression Paste ${ }^{\mathrm{R}}$ ] at point 1, 2, 3, and 4 width was 10.95, 10.44, 11.16 and 10.67 respectively. Calculated Coefficient of Variance value with Viscogel tissue conditioner is $0.70 \%$ and with zinc oxide eugenol paste $1.04 \%$.

\section{CONCLUSION}

With these results and statistical analysis, with $5 \%$ of level of significance (as alpha $=0.05$ ) i.e. $95 \%$ confident, we found enough evidence to conclude that usage of Viscogel tissue conditioner is more effective and accurate than zinc oxide eugenol impression paste in neutral zone technique.

\section{KEY WORDS}

Neutral Zone, Denture Stability, Tissue Conditioner, Zinc Oxide Eugenol Paste, Resorbed Mandibular Ridge
Corresponding Author: Dr. Nihit Singh Rana, A-10/14, MIG Ved Nagar, Opp. Deshmukh Hospital, Ujjain, Madhya Pradesh, India. E-mail:dr.nihit.singh@gmail.com

DOI: $10.14260 / \mathrm{jemds} / 2020 / 107$

Financial or Other Competing Interests: None.

How to Cite This Article:

Rana NS. A clinical comparative study of two different materials used in recording neutral zone in completely edentulous patients at Jaipur dental college, Jaipur. J. Evolution Med. Dent. Sci. 2020;9(8):473478, DOI: $10.14260 /$ jemds/2020/107

Submission 27-03-2019,

Peer Review 30-01-2020,

Acceptance 06-02-2020,

Published 24-02-2020. 


\section{BACKGROUND}

"All of the good that is built into a denture is rather useless to a patient unless he can keep it in position during ordinary oral functions." Complete dentures are primarily mechanical devices, but since they function in the oral cavity, they must be fashioned so that they are in harmony with normal neuromuscular function. When all of the natural teeth have been lost, there exists within the oral cavity a void which is the potential denture space. The neutral zone is that potential denture space where the forces of the cheeks and lips pressing outward are neutralised by the forces of the cheeks and lips pressing the denture inward. ${ }^{1}$ Natural teeth are also arranged in that potential space, the neutral zone. The concept for fabricating stable dentures, A NEUTRAL ZONE was first introduced by Sir E. Wilfred Fish in 19331,2,3.

Importance of Neutral zone is realised by dentist in rehabilitating patients with resorbed residual alveolar ridges specially the mandibular arch. It is not only the treatment of choice in resorbed ridges but also in patients with partial glossectomy, ${ }^{4}$ mandibular resection or motor nerve damage to tongue. 5 There are many techniques available for recording neutral zone in which movement such as sucking, swallowing, whistling and pursuing of the lips have been used. A large number of material such as wax, impression compound, silicone, resilient lining material and polymer of dimethyl siloxane filled with calcium silicate have been used. In this concept tooth position and flange are determined by the compound occlusal rims that results from functional neuromuscular co-ordination of muscle action. The accurate recording is carried out by an easily flowing material such as tissue conditioner or zinc oxide eugenol paste which is placed on the external surface of the trial denture after scraping away the wax between neck of teeth and periphery.

Tissue conditioning materials are manufactured as powder and liquid. Powder is mainly an acrylic resin and is mixed with oily liquid plasticizer (generally an alcoholic solvent) in a proportion of 1.25 to 1.5 of powder to 1 of liquid. This results information of soft and elastic gel, basically it's a relining material but we can also use it as impression material. ${ }^{6}$ The zinc oxide-eugenol impression pastes have been introduced in 1930's and have become increasingly popular in impression procedures in denture prosthesis. Before this time, mixtures of zinc oxide and eugenol were used as dental cements. ${ }^{7}$ These two are commonly available materials but still there is a great confusion among clinicians about choosing the best material for recording Neutral zone.

The present study was undertaken in remote area to study the accuracy of reproducibility of Neutral Zone by two commonly available dental materials. Materials selected were Viscogel tissue conditioner and zinc oxide. eugenol paste with certain modification to facilitate accurate recording. Aim in selecting zinc oxide Paste was to evaluate the accuracy of its records to enable standardisation of the recording with cheaper material to enable a large number of extremely poor patients to obtain maximum benefits.

\section{METHODS}

Viscogel tissue conditioner and zinc oxide eugenol paste are easy flowing recording materials and can be recorded by using support. Hence to study the accuracy of the reproducibility of the neutral zone, specially designed acrylic rims with occlusal stops were used which supported the recording materials adequately in the posterior region of the inter ridge space. Swallowing method was used to record the neutral zone, five times by each material. Bucco-lingual dimension of the neutral zone was recorded at predetermined reference points for all individuals with the help of a micrometre. 40 patients were selected with Class 1 jaw relations.

\section{Patients}

1. Selected patients were between the age group of 45 to 65 years.

2. The individuals were free from oral pathological lesions and major systemic problems.

3. Selected patients had average inter arch space present.

4. Patients with abnormal tongue size were excluded in this study.

5. The jaw relation was skeletal class I type.

6. It was also ensured that no flabby ridge existed at the time of experiments.

7. Patient have adequate salivary flow.

8. Both the male and female patients were included in the study.

9. Patients with neurological disorder were not included in the study.

\section{Fabrication of Compound Occlusal RIMS}

Special occlusal rims were constructed for the study by sequentially following the steps mentioned below. Initially primary impressions of the denture bearing tissues were made in stock trays. Upper and lower final impressions were made with zinc oxide eugenol impression paste [DPI Impression Paste ${ }^{\mathrm{R}}$ ] with the help of custom acrylic trays. Stone models were prepared from upper and lower final impressions. Undercuts were blocked and cold mould seal was applied using brush. 2 sets of denture bases for the record blocks were made with the help of clear acrylic resin [DPI-RR cold cure] of $1.5 \mathrm{~mm}$ thickness with the help of stone templates. The acrylic resin is allowed to polymerize in the pressure pot with water bath at $37^{\circ} \mathrm{C}$ and 20 psi for 15 minutes $^{8}$ and bases were trimmed, polished and smoothened. ${ }^{9}$ On the upper model below mentioned indelible points were marked with marker for future reference. Incisive papilla's most distal point was marked in the centre and two pterygomaxillary notches were posteriorly marked on both sides. The ridge was marked by a line passing antero-posteriorly at its highest part. A horizontal line was drawn from the distal end of the incisive papilla, towards the ridge. From the point on the ridge where the horizontal line from the papilla meet the antero-posterior line on the ridge, a point 3 centimeters away, towards maxillary tuberosity was marked on both sides of the ridge. These two marks on the crest of the ridge were transferred to the clear acrylic resin denture bases. Next wax occlusal rims were built on both upper and lower acrylic base plates. 
Tentative jaw relations were recorded, and models were articulated on Wide view Hanau semi-adjustable arcon type articulator. Wax was cut away between anterior and posterior marks from the acrylic denture base to create windows for the neutral zone recording medium from both upper as well as lower record blocks. Then wax rims were also replaced by compound rims [Y-DENTS ${ }^{\mathrm{R}}$ ] of $2 \mathrm{~mm}$ thickness and height same as the wax rims. In the space of $3 \mathrm{~cm}$ window, a stainless steel wire (19 gauge) with $\mathrm{u}-\mathrm{v}$ loop was also inserted to stabilize the recording material, also junctions of wire and compound were stabilized by acrylic resin so upper compound rims left at three places and called as centric stops and the similar stops were formed in the lower record rim by relating them with the upper rim in the patient's mouth. In the upper centric stops V shaped projections in the Centre and V shaped groove in the lower centric stops were made, which stabilized the record blocks, when patients carried out muscle movements to shape the recording medium intra-orally. Again check the denture bases along with compound rims in patient mouth to verify the jaw relation.

\section{Manipulation of Material-Viscogel Tissue Conditioner}

The materials used in this study were zinc oxide-eugenol dental impression paste [DPI Impression Paste ${ }^{\mathrm{R}}$ ] and tissue conditioner [Dentsply, Visco-gel, Temporary Soft Denture Liner]. Ethyl methacrylate powder of tissue conditioner was mixed with its liquid, butyl phthalate butyl glycol ate ester in ethanol for 30 seconds as per manufacturer's directions in the mixing vessel supplied. The material after 2.5 minutes was loaded on the acrylic resin denture bases to fill up previously created window.

\section{Recording of Neutral Zone}

The patient was told to close in centric relation and wait for 3 minutes. After this patient was instructed to carry out swallowing, sipping, whistling, sucking and check movements with mouth closed for approximately 1 hours, once a minute..$^{10}$ After moulding of the tissue conditioner, at the established vertical jaw relation, the rims were removed from the patient's mouth. When the final "moulded" rims were returned to the articulator then with a marker, five vertical lines were drawn between both the upper and lower casts buccally as well as lingually. Five horizontal lines were also drawn antero-posteriorly dividing the inter-arch distance into four equal parts by the use of graph paper.

The Lines were named as -

a. Horizontal Lines: (i) Upper base plane line (ii) Upper median plane line. (iii) Occlusal plane line (iv) Lower median plane line (v) Lower base plane line.

b. Vertical lines: (i) Anterior vertical line. (ii) Anterior intermediate vertical line. (iii) Central vertical line. (iv) Posterior intermediate vertical line. (v) Posterior vertical line.

Four points were selected and the recorded as reference points common in all the patients-

- Point 1- Situated at the junction of horizontal Upper median plane line and anterior intermediate vertical plane line.

- $\quad$ Point 2- Situated at the junction of Upper median plane line and posterior intermediate vertical plane line.
- $\quad$ Point 3- Situated at the junction of lower median plane line and anterior intermediate vertical plane line.

- Point 4- Situated at the junction of lower median plane line and posterior intermediate vertical plane line.

A point micrometer (range $0-25 \mathrm{~mm}$ ) was used. Buccolingual width was measured at four predetermined and marked points, on the material which was moulded into proper shape by the tongue as well as cheeks. Each of the above mentioned procedure was carried out 5 times for each individual. Individuals were made to practice with the materials few times before accurate impressions were obtained to proceed further in this study.

\section{Manipulation of Material- zinc oxide eugenol Impression Paste}

zinc oxide eugenol impression paste [DPI Impression Paste ${ }^{\mathrm{R}}$ ] was used as the other material in this study with a little modification to prolong the setting time. Setting time of the zinc oxide eugenol paste was prolonged by the addition of $1 \mathrm{ml}$ of olive oil, ${ }^{10}$ to an equal length of 10 centimetres of base and catalyst paste. This not only prolonged the setting time but also reduced the rigidity of material that helped in more accurate recording of neutral zone. ${ }^{11}$ Additional steps taken, in addition to the use of olive oil in prolonging setting time were to cool the mixing spatula and mixing slab in refrigerator for half an hour just before use and then mix the paste within 30 seconds.

\section{Recording the Neutral Zone}

The mixed paste was quickly applied on the denture base with compound rims in the window and taken into the mouth, the same movements were carried out by the patient as were done with the tissue conditioner. Compound rims with denture bases were removed after 20 minutes and again adapted back to the articulator. Markings were made in a similar way and point micrometer range $0-25 \mathrm{~mm}$ was used to measure the bucco-lingual width at the same at four predetermined and marked points, on the material which was moulded. Similar procedure was also carried out 2 times in each patient. After recording neutral zone patients were followed by try-in procedure and complete denture were fabricated and inserted.

\section{RESULTS}

The data obtained as a result of the experiments has been presented in various tables. The descriptive statistics for the obtained data, as a result of the experiments on patients, has been presented in the following table (Table 1). Table 1 consolidates all the observations made for the neutral zone dimension (expressed in millimetres) during the experiments for all reference points by using viscogel tissue conditioner and zinc oxide eugenol impression paste. The last row shows the result of performed paired t-test, with 39 degree of freedom, which allows us to the compare the size of difference between the means of the sample obtained based on our cases performed. 


\section{Note}

All the collected sample series data is tested in Normal Quant by which we can conclude that sample data do not give sufficient evidence to warrant rejection of the claim that these values come from a normal population.

\begin{tabular}{|c|c|c|c|c|c|c|c|c|}
\hline \multirow{3}{*}{$\begin{array}{c}\mathrm{X} 1 \text { for } \mathrm{ZE}^{*} \\
\mathrm{X} 2 \text { for } \mathrm{VG}^{* *}\end{array}$} & \multicolumn{8}{|c|}{ Reference Points Number } \\
\hline & \multicolumn{2}{|c|}{ Point 1} & \multicolumn{2}{|c|}{ Point 2} & \multicolumn{2}{|c|}{ Point 3} & \multicolumn{2}{|c|}{ Point 4} \\
\hline & $\mathbf{Z E}^{*}$ & VG** & $\mathbf{Z E}^{*}$ & $\mathrm{VG}^{* *}$ & $\mathbf{Z E}^{*}$ & $\mathbf{V G}^{* *}$ & ZE* & $\mathbf{V G}^{* *}$ \\
\hline No. of patients & 40 & 40 & 40 & 40 & 40 & 40 & 40 & 40 \\
\hline Min & 8.53 & 8.04 & 7.91 & 7.62 & 8.74 & 8.15 & 8.15 & 7.81 \\
\hline Max & 13.51 & 13.06 & 13.07 & 12.76 & 13.7 & 13.29 & 13.23 & 12.97 \\
\hline Mean & 10.95 & 10.36 & 10.44 & 10.06 & 11.16 & 10.51 & 10.67 & 10.21 \\
\hline Standard Deviation & 1.45 & 1.32 & 1.47 & 1.34 & 1.43 & 1.31 & 1.46 & 1.34 \\
\hline $\begin{array}{c}\text { Mean of difference } \\
(\mathrm{X} 1 \text { - X2) }\end{array}$ & \multicolumn{2}{|c|}{0.59} & \multicolumn{2}{|c|}{0.38} & \multicolumn{2}{|c|}{0.65} & \multicolumn{2}{|c|}{0.46} \\
\hline SD of difference (s) & \multicolumn{2}{|c|}{0.39} & \multicolumn{2}{|c|}{0.30} & \multicolumn{2}{|c|}{0.44} & \multicolumn{2}{|c|}{0.30} \\
\hline SE of mean (se) & \multicolumn{2}{|c|}{0.06} & \multicolumn{2}{|c|}{0.05} & \multicolumn{2}{|c|}{0.07} & \multicolumn{2}{|c|}{0.05} \\
\hline t value - calculated & \multicolumn{2}{|c|}{9.63} & \multicolumn{2}{|c|}{8.16} & \multicolumn{2}{|c|}{9.24} & \multicolumn{2}{|c|}{9.60} \\
\hline
\end{tabular}

\begin{tabular}{|c|c|c|c|c|c|c|c|c|}
\hline \multirow[t]{2}{*}{ 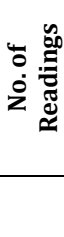 } & \multicolumn{2}{|c|}{ 采 } & \multirow[t]{2}{*}{ के } & \multirow{2}{*}{ 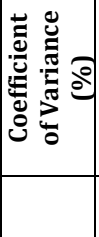 } & \multicolumn{2}{|l|}{ 卷 } & \multirow[t]{2}{*}{ के } & \multirow{2}{*}{ 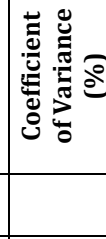 } \\
\hline & \begin{tabular}{|l|} 
Width \\
in $\mathbf{~ m m}$ \\
\end{tabular} & Mean & & & \begin{tabular}{|c|} 
Width in \\
mm
\end{tabular} & Mean & & \\
\hline 1 & \begin{tabular}{|c|}
10.63 \\
\end{tabular} & \multirow{10}{*}{10.71} & \multirow{10}{*}{0.112} & \multirow{10}{*}{1.04} & 10.23 & \multirow{10}{*}{10.29} & \multirow{10}{*}{0.07} & \multirow{10}{*}{0.70} \\
\hline 2 & 10.87 & & & & 10.37 & & & \\
\hline 3 & 10.64 & & & & 10.26 & & & \\
\hline 4 & 10.58 & & & & 10.19 & & & \\
\hline 5 & 10.89 & & & & 10.41 & & & \\
\hline 6 & 10.73 & & & & 10.30 & & & \\
\hline 7 & 10.59 & & & & 10.20 & & & \\
\hline 8 & 10.76 & & & & 10.31 & & & \\
\hline 9 & 10.66 & & & & 10.27 & & & \\
\hline 10 & 10.79 & & & & 10.34 & & & \\
\hline \multicolumn{9}{|c|}{ atistics } \\
\hline
\end{tabular}
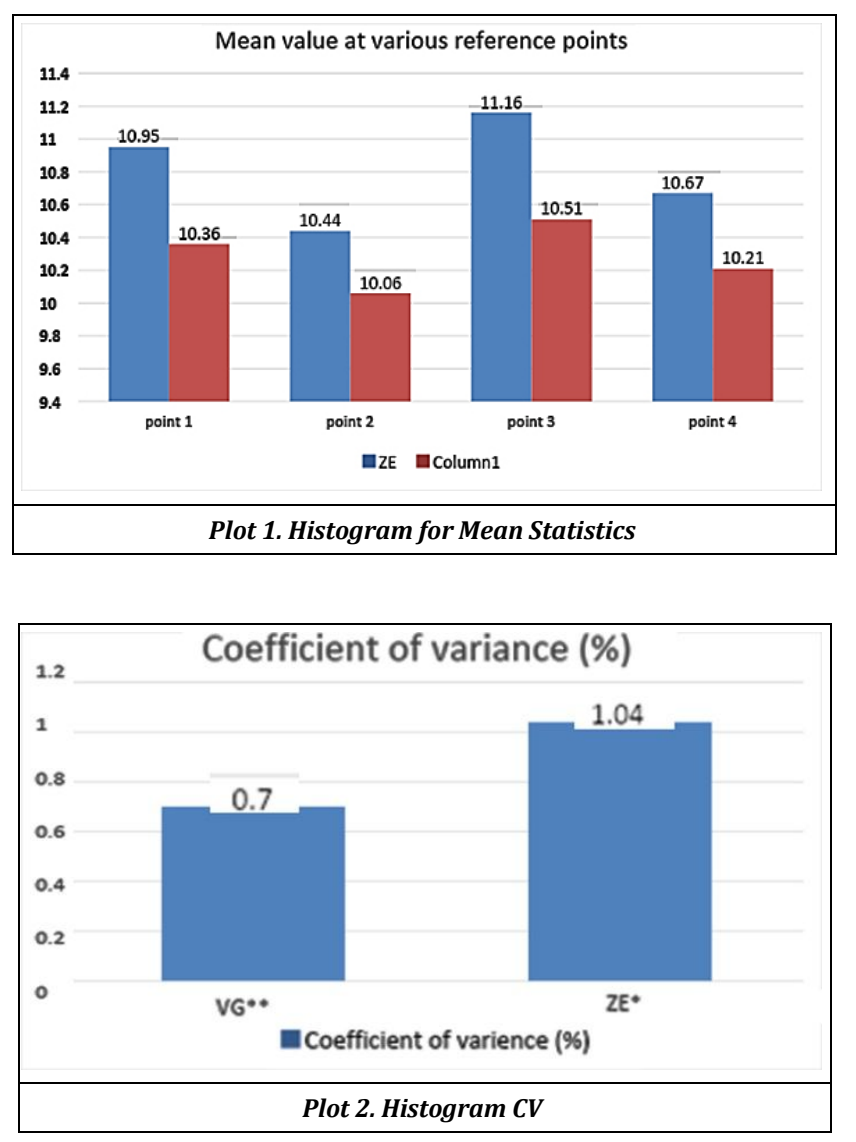

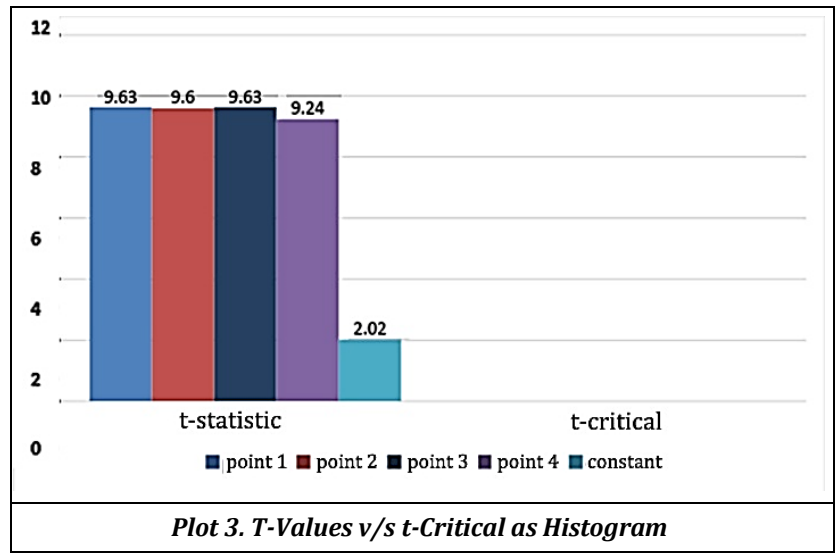

Next, we show the histogram of the mean width (in mm.) of the neutral zone at reference points 1,2, 3, and 4 for tissue conditioner [Dentsply, Visco-gel, Temporary Soft Denture Liner], whose values are 10.36, 10.06, 10.51 and 10.21 respectively. However, for the zinc oxide eugenol impression paste [DPI Impression Paste R] these values, at reference points $1,2,3$, and 4, respectively, are $0.95,10.44,11.16$ and 10.67. In Table 2 and Plot 2 we summarize and compare using histogram the calculated Coefficient of Variation (CV), in percentage, which measures the dispersion of the recorded data. Calculated $\mathrm{CV}$ values with viscogel tissue conditioner and zinc oxide eugenol impression paste are, respectively, $0.70 \%$ and with $1.04 .^{*}$ : zinc oxide-eugenol impression paste [DPI Impression Paste R] **: Tissue conditioner [Dentsply, Viscogel, and Temporary Soft Denture Liner].

We conclude this section by comparing in plot the calculated $t$-values, by employed paired t-test, for 2 different materials at each reference point 1, 2, 3 and 4. In precise the values are 9.63, 9.60, 9.63 and 9.24 respectively. Note that these values are greater than t-critical value (2.02) at alpha 0.05 with 39 degrees of freedom.

\section{DISCUSSION}

For the muscular forces to be stabilizing in nature, the denture must be so constructed that they will receive these forces at proper angle and the resultant forces becomes neutral and maintain the denture in its position. The coordination of complete dentures with forces and neuromuscular function is the foundation of successful, stable dentures. The neutral zone is that potential space for stable denture. Neutral zone technique has been criticized based on the claim that it is supported by empirical evidence. However, other authors maintain that is inaccurate as neutral zone technique is based on the significant clinical observation on the role of destabilizing forces the muscles apply to complete dentures during functional movements. Furthermore, the large number of case reports accumulated in a short period of time and clinical studies conducted by Fahmy \& Kharat ${ }^{12}$, Zaigham AM and Mahesh $\mathrm{S}^{13}$, Sudhindra M, Shrinivas $\mathrm{P}$ and Girish and Sharif $\mathrm{M}^{7}$, Azad AA and Ahmed $\mathrm{S}^{14}$ undermine this criticism and add to the validity of Neutral zone technique. They found improved comfort and speech clarity reported by patients upon wearing complete denture fabricated using neutral zone technique when compared to conventional complete denture. 
For recording neutral zone Trench et al ${ }^{15}$ were the first who has proposed the use of impression compound. Although this advice is widely followed and other materials like tissue conditioner, wax, zinc oxide eugenol impression material, chairside relining material, acrylic resins and silicone materials has been used widely in various studies.

The present study was carried to compare the reproducibility of two dental material-the tissue conditioner and zinc oxide eugenol impression paste. Karlson S Hedegard B and Odont ${ }^{16}$, Razek MKA and Abdalla F9, Fahmy FM17, Ohkubo C, Hanatani S, Hosoi T and Mizuno Y18, Gahan MJ and Walmsy $\mathrm{AD}^{19}$, and Kursoglu P, Nilgun Calikkocaoglu S20 suggested the use of tissue conditioner for recording neutral zone and as it is mucostatic, odourless and tasteless and have long standing time so preferred over zinc oxide euginol paste But according to Krammer RV and Dentista $\mathrm{C}^{6}$ using tissue conditioner for recording impression is controversial

Whereas Schiesser $\mathrm{FJ}^{2}$ suggested the use of zinc oxide eugenol impression paste even though it irritates the patient's mucosa. In this study Special occlusal rims of impression compound ( $2 \mathrm{~mm}$ width) were fabricated after jaw relation and mounting of casts on the articulator, on the 2 sets of selfcure clear acrylic denture bases (1.5 mm thickness). Clear acrylic was used in this study so that markings on the casts (line marked horizontal to the distal end of incisive papilla and $3 \mathrm{~cm}$ distal to that horizontal line) can be easily transferred on to the acrylic denture bases and easily fabricate compound occlusal rims up to those markings accurately.

Recorded data has been tabulated and Table 1 infers that the width (in mm.) of the neutral zone is minimum at the posterior points that is at point 2 and 4 . It is 10.44 for zinc oxide eugenol impression paste and 10.06 for viscogel tissue conditioner (mean values) at point 2 and 10.67 for zinc oxide eugenol impression paste and 10.21 for viscogel tissue conditioner at point 4 . It increases towards the anterior points that is at point 1 and 3 . It is 10.95 for zinc oxide eugenol impression paste and 10.36 for viscogel tissue conditioner (mean values) at point 1 and 11.16 for zinc oxide eugenol impression paste and 10.51 for viscogel tissue conditioner (mean values) at point 3 . This data is similar to the data provided by Razek MKA and Abdalla F (1981) ${ }^{9}$ because of action of buccinator muscle and the convexity of the lateral borders of the tongue. So, the freedom for molar placement is less than premolars and anterior teeth.

Descriptive statistics and inference statistical test for observation made at reference point 1,2,3 and 4 are tabulated in Table 1. Here descriptive includes the calculation of mean (arithmetic) and dispersion (standard deviation) in the difference of the recorded data, which were calculated as $0.59 \pm 0.39,0.38 \pm 0.30,0.65 \pm 0.44 \wedge 0.46 \pm 0.30$,

respectively. The variability of the sample mean in the difference data was calculated as the standard error of the mean (SE); we have calculated this parameter to quantify how precisely we know the true mean of the population. Estimated values of SE were $0.06,0.05,0.07 \wedge 0.05$, respectively, these small values of SE infers that mean of the sample is likely to be closer to the true mean of the population (set of all the patients required neutral zone technique in the near area) i.e. we can conclude that these observed mean values are good approximation for the correct value which we expect in the application of neutral zone technique on a randomly chosen patient

In this study we had recorded neutral zone on same patient twice with two different materials, with tissue conditioner [Dentsply, Visco-gel, and Temporary Soft Denture Liner] and with zinc oxide-eugenol impression paste [DPI Impression Paste ${ }^{\mathrm{R}}$. To test the null hypothesis of same mean width values for both material in the sample we have used paired t-test, this decision is supported by statistical theory. For the observed data at each reference point 1, 2, 3 and 4 in which estimated t-values calculated were 9.63, 9.60, 9.63 and 9.24 respectively. These values were greater than $\mathrm{t}$-critical value (2.02) at alpha 0.05 with 39 degrees of freedom. As per the theory, if absolute value of $\mathrm{t}$-value $>$ absolute value of $\mathrm{t}$ critical then we can reject the null hypothesis and according to the results $t$-value is greater so we can conclude that there is statistically difference between the two populations mean i.e. above paired t-test (statistical) results shows that the mean widths, in the neutral zone in the treatment with zinc oxide eugenol impression paste and next with viscogel tissue conditioner, differs significantly.

To compare the effectiveness and accuracy of the two materials, we have obtained 10 sample points data at a fixed reference point 1 from one individual with repeated application of both materials. The observed data points were tabulated in Table 6 with the calculation of, standard deviation and coefficient of variation statistic and we can observe that the mean and standard deviation value of width of neutral zone with zinc oxide eugenol impression paste $(10.71,0.112$ $\mathrm{mm}$ respectively) is greater than the mean value with viscogel tissue conditioner (10.29, $0.07 \mathrm{~mm}$ respectively).

By previous tables data we have concluded the sample point for both materials are not from same population (as mean is statistically different) so to compare the dispersion in probability distribution of sample data from different population we have calculated another statistic known as coefficient of variation (CV); it measures the spread of a data set as a proportion of its mean and expressed as a percentage. In the table the calculated value of $\mathrm{CV}$ with zinc oxide eugenol paste $(1.04 \%)$ is greater than $\mathrm{CV}$ value with viscogel tissue conditioner $(0.70 \%)$ i.e. the spread in the sample data, collected by repeated procedure in one individual, of the width of neutral zone with zinc oxide eugenol impression paste [DPI Impression Paste ${ }^{\mathrm{R}}$ ] is more than with viscogel tissue conditioner [Dentsply, Visco-gel, Temporary Soft Denture Liner]. This is consistent with the expectation of the superiority (in the sense of close values under repeated usage) in the usage of viscogel tissue conditioner in neutral zone technique as compared to zinc oxide eugenol impression paste. The study done by Gursharan Singh (2011) ${ }^{10}$ supports this conclusion that viscogel tissue conditioner is better material then zinc oxide eugenol impression paste as the less the bucco-lingual width, more is the accuracy and less the coefficient of variation, less is the spread so better the material. We are able to conclude the above statement because we had chosen same patient and similar denture bases and occlusal rims (in terms of material, height and width), only recording material were different. 


\section{CONCLUSIONS}

Usage of viscogel tissue conditioner is more effective and accurate than zinc oxide eugenol impression paste in neutral zone technique.

\section{REFERENCES}

[1] Wright CR. Evaluation of the factors necessary to develop stability in mandibular dentures. J Pros Dent 1966;16 (3):414-30.

[2] Schiesser FJ. The neutral zone and the polished surfaces in complete denture. J Pros Dent 1964;14 (5):854-65.

[3] Beresin VE, Schiesser FJ. The neutral zone in complete and partial dentures. Chap - 1 and 2. $2^{\text {nd }}$ edn. Mosby 1978.

[4] Wee AG, Cwynar RB, Cheng AC. Utilization of the neutral zone technique for maxillofacial patients. J Prosthod 2000;9 (1):2-7.

[5] Porwal A, Jain P, Biradar SP, et al. Neutral zone approach for rehabilitation of severely atrophic ridge. International Journal of Dental Clinics 2010;2 (3):53-7.

[6] Von Krammer R, Cirupano-Dentista. Tissue conditioners. J Pros Dent 1971;25 (3):244-50.

[7] Mahesh S, Sudhindra M, Sreenivas P, et al. Comparative study to evaluate masticatory efficiency speech and comfort using dentures fabricated with conventional method and neutral zone concept. BFUDJ 2011;2 (1):114.

[8] O'Toole TJ, Furnish GM, Von Fraunhofer JA. Linear distortion of acrylic resin. Journal of Prosthetic Dentistry 1985;53 (1):53-5.

[9] Razek MKA, Abdalla F. Two-dimensional study of neutral zone at different occlusal vertical heights. J Pros Dent 1981;46 (5):484-9.
[10] Singh G. A clinical evaluation of neutral zone in complete denture patients. BFUDJ 2011;2 (1):15-9.

[11] Makzoume JE. Morphology comparison of two neutral zone impression technique: a pilot study. J Pros Dent 2004;92 (6):563-8.

[12] Fahmy FM, Kharat DU. A study of the importance of the neutral zone in complete dentures. J Pros Dent 1990;64 (4):459-62.

[13] Zaigham AM. A comparative study of selective pressure impression technique and neutral zone approach in a trophic mandibular ridges. J Pros Dent 2006;26 (2):24750.

[14] Sharif M, Azad AA, Ahmed S. Comparison of patient's satisfaction level with complete dentures fabricated by neutral zone technique and conventional technique. Pakistan Oral and Dental Journal 2013;33 (1):187-91.

[15] Porwal A, Sasaki K. Current status of neutral zone: a literature review. The Journal of Prosthetic Dentistry 2013;109 (2):129-34.

[16] Karlsson S, Hedegard B, Odont. A study of the reproducibility of the functional denture space with a dynamic impression technique. J Pros Dent 1979;41 (1):21-5.

[17] Fahmi FM. The position of the neutral zone in relation to the alveolar ridge. J Pros Dent 1992;67 (6):805-9.

[18] Ohkubo C, Hanatani S, Hosoi T, et al. Neutral zone approach for denture fabrication for a partial glossectomy patient: a clinical report. J Pros Dent 2000;84 (4):390-3.

[19] Gahan MJ, Walmsley AD. The neutral zone impression revisited. British Dental Journal 2005;198 (5):269-72.

[20] Kursoglu P, Ari N, Calikkocaoglu S. Using tissue conditioner material in neutral zone technique. N Y State Dent J 2007;73 (1):40-2. 